\title{
Achado Incomum de Rara e Exuberante Xantomatose em Caso de Hiperlipidemia
}

\author{
Unusual Finding of Rare Exuberant Xanthomatosis in Hyperlipidemia
}

Enrico Manfredini ${ }^{10}$ e Renato Jorge Alves ${ }^{1,2}$ (1)

Faculdade de Ciências Médicas da Santa Casa de São Paulo (FCMSCSP), ${ }^{1}$ São Paulo, SP - Brasil

Irmandade da Santa Casa de Misericórdia de São Paulo - Departamento de Medicina, ${ }^{2}$ São Paulo, SP - Brasil

\section{Introdução}

Hiperlipidemias podem aumentar a morbimortalidade. Foram classificadas por Fredrickson em fenótipos: I, Ila, IIb, III, IV e V. ${ }^{1-5}$ Nas mistas, há hipercolesterolemia e hipertrigliceridemia, fenótipos IIb e III, com colesterolemia e trigliceridemia de 250 a $300 \mathrm{mg} / \mathrm{dL}$ no fenótipo $\mathrm{Ilb}$, e 500 a $600 \mathrm{mg} / \mathrm{dL}$ ou mais, no III, respectivamente. É incomum o aparecimento de pancreatite em ambos, bem como xantomatose no llb. Xantomas e complicações cardiovasculares são mais frequentes no fenótipo III. 2,6

Apresentamos um caso de hiperlipidemia com relevantes alterações lipídicas, pancreatite e exuberante xantomatose.

\section{Relato do Caso}

Masculino, 48 anos de idade, natural de Manaus, comerciante, com histórico de pancreatite hemorrágica (2004), hipertensão arterial e diabetes tipo 2 desde 2006, retinopatia hipertensiva grau 3 e retinopatia diabética proliferativa grave. Negava tanto histórico familiar de doenças cardiovasculares ou dislipidemias quanto consanguinidade na família. Usava enalapril $10 \mathrm{mg} / \mathrm{dia}$, dapagliflozina $5 \mathrm{mg} /$ dia, metformina $1.000 \mathrm{mg} / \mathrm{dia}$, gliclazida $120 \mathrm{mg} / \mathrm{dia}$ e insulina NPH 16Ul/dia. Negava uso prévio de estatina, apenas fibrato irregularmente.

Assintomático e anictérico. Peso: 89 kg, altura: $172 \mathrm{~cm}$, IMC: $30,1 \mathrm{~kg} / \mathrm{m}^{2}$, pressão arterial: 120/90 mmHg, frequência cardíaca: 80 bpm. Pulmões limpos, bulhas rítmicas normofonéticas, sopro protossistólico em área aórtica $2 / 6+$, sem sopros carotídeos. Pulsos distais regulares e sem alterações. Abdome globoso, com cicatriz xifoumbilical. Membros inferiores sem edema.

Destacava-se a presença de múltiplas e extensas lesões nodulares, indolores, em cotovelos, articulações

\section{Palavras-chave}

Hiperlipidemias; Dislipidemias; Xantomatose; Hipolipemiantes.

\section{Correspondência: Renato Jorge Alves •}

Faculdade de Ciências Médicas da Santa Casa de São Paulo (FCMSCSP) Rua Dr. Cesário Mota Júnior, 112. CEP 01221-020, Santa Cecilia, São Paulo, SP - Brasil

E-mail: renatoalves178@gmail.com

Artigo recebido em 17/09/2020, revisado em 04/02/2021, aceito em 24/02/2021

DOI: https://doi.org/10.36660/abc.20200999 metacarpofalângicas e interfalângicas bilateralmente, joelhos e tornozelos, compatíveis com xantomas tuberosos e tendinosos (Figura 1). Não apresentava xantoma estriado palmar.

Foi realizada eletroforese de lipoproteínas: fração alfa 6,2\%, beta e pré-beta 93,8\%, compatível com fenótipo IIb.

Devido ao quadro da hiperlipidemia, optou-se pela introdução de atorvastatina 40, após 6 meses, 80 mg/dia e ciprofibrato $100 \mathrm{mg} / \mathrm{dia}$, associados a modificações do estilo de vida e dietoterapia. Após essa terapêutica, houve regressão significativa das lesões xantomatosas (Figura 2) e da hiperlipidemia (Tabela 1).

\section{Discussão}

Trata-se de achado incomum de xantomatose difusa em paciente com fenótipo IIb, que costuma migrar para Ila e IV na prática clínica.

Essa xantomatose é raramente vista no fenótipo IIb, principalmente a forma tuberosa em tendão de Aquiles, mais encontrada em hipercolesterolemia familiar (HF). ${ }^{7}$ O ecocardiograma evidenciou calcificação da válvula aórtica, achado também em casos graves de HF ou de elevação plasmática de lipoproteína(a) (Lp[a]).8,9 No entanto, uma resposta muito satisfatória à terapia com estatina, como ocorreu nesse caso, não seria comum na $\mathrm{HF}$, principalmente na forma homozigótica. ${ }^{1,3}$

A hipertrigliceridemia acentuada indicaria fenótipo IV ou V; contudo, a eletroforese de lipoproteínas mostrou elevações das frações beta e pré-beta. ${ }^{6}$ Entretanto, hipertrigliceridemia $>1.500 \mathrm{mg} / \mathrm{dL}$ com xantomas tuberosos e tendinosos seria compatível com dislipidemia mista. ${ }^{2,6}$

No fenótipo III, além de xantomatose tuberosa e eruptiva, haveria xantomatose palmar e doença aterosclerótica precoce. ${ }^{9}$ Ainda, as concentrações plasmáticas de colesterol e triglicérides seriam muito elevadas, mas quase similares. Entretanto, pelo fato de os distúrbios metabólicos terem contribuído para o agravamento do quadro clínico e os xantomas se assemelharem à xantomatose tuberoeruptiva, disbetalipoproteinemia (tipo III) associada a defeitos genéticos, como HF ou elevação de Lp(a), seria a hipótese apropriada a ser considerada.

Outras hipóteses excluídas seriam: xantomatose cerebrotendinosa - não há alterações neurológicas, ${ }^{10,11}$ e sitosterolemia, devido à resposta satisfatória com estatina, ${ }^{12,13}$ embora pudesse ser excluída por genotipagem.

É relevante relatar a ocorrência de pancreatite aguda em 2004, com consequente diabetes, mais frequente 

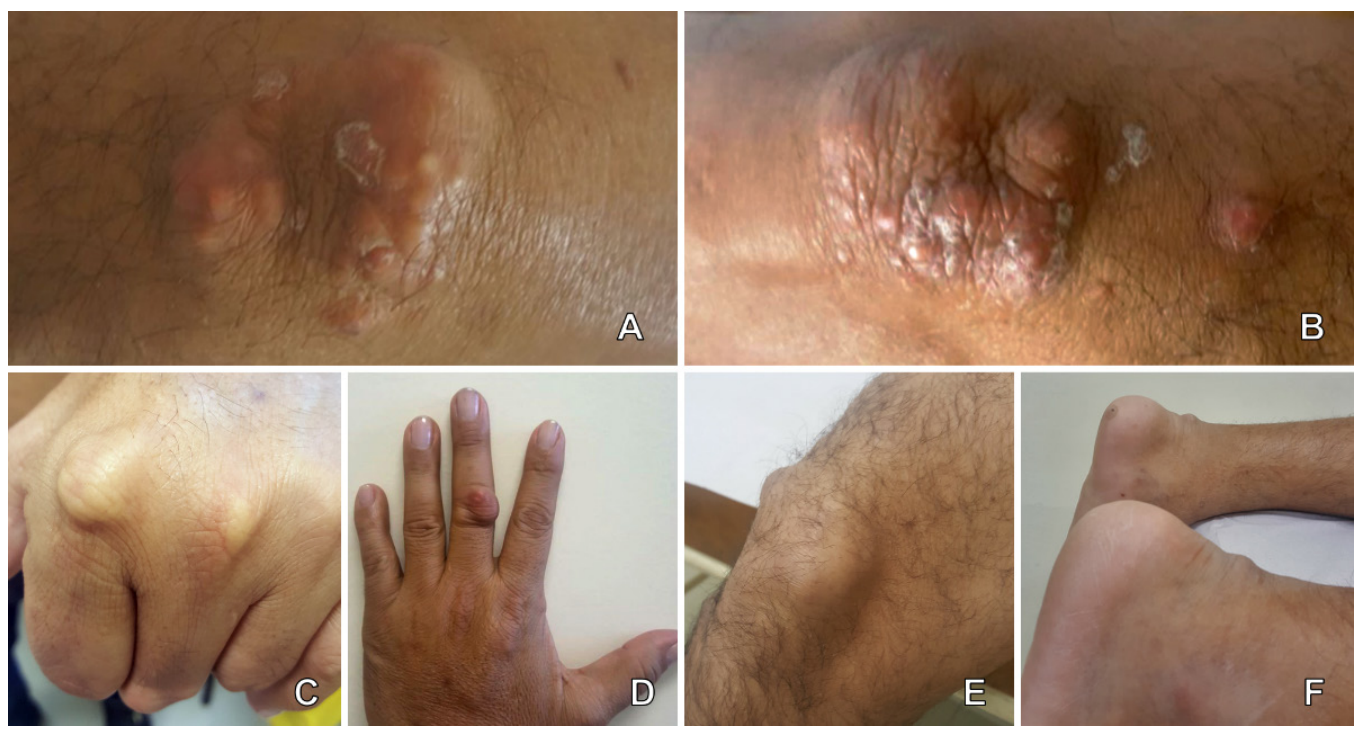

Figura 1 - Xantomatose prévia. A) Cotovelo direito. B) Cotovelo esquerdo. C) Segunda articulação metacarpofalângica esquerda. D) Terceira articulação interfalângica proximal direita. E) Joelho direito. ) Ambos os tendões de Aquiles. Fonte: imagens obtidas pelos autores durante consulta de rotina.

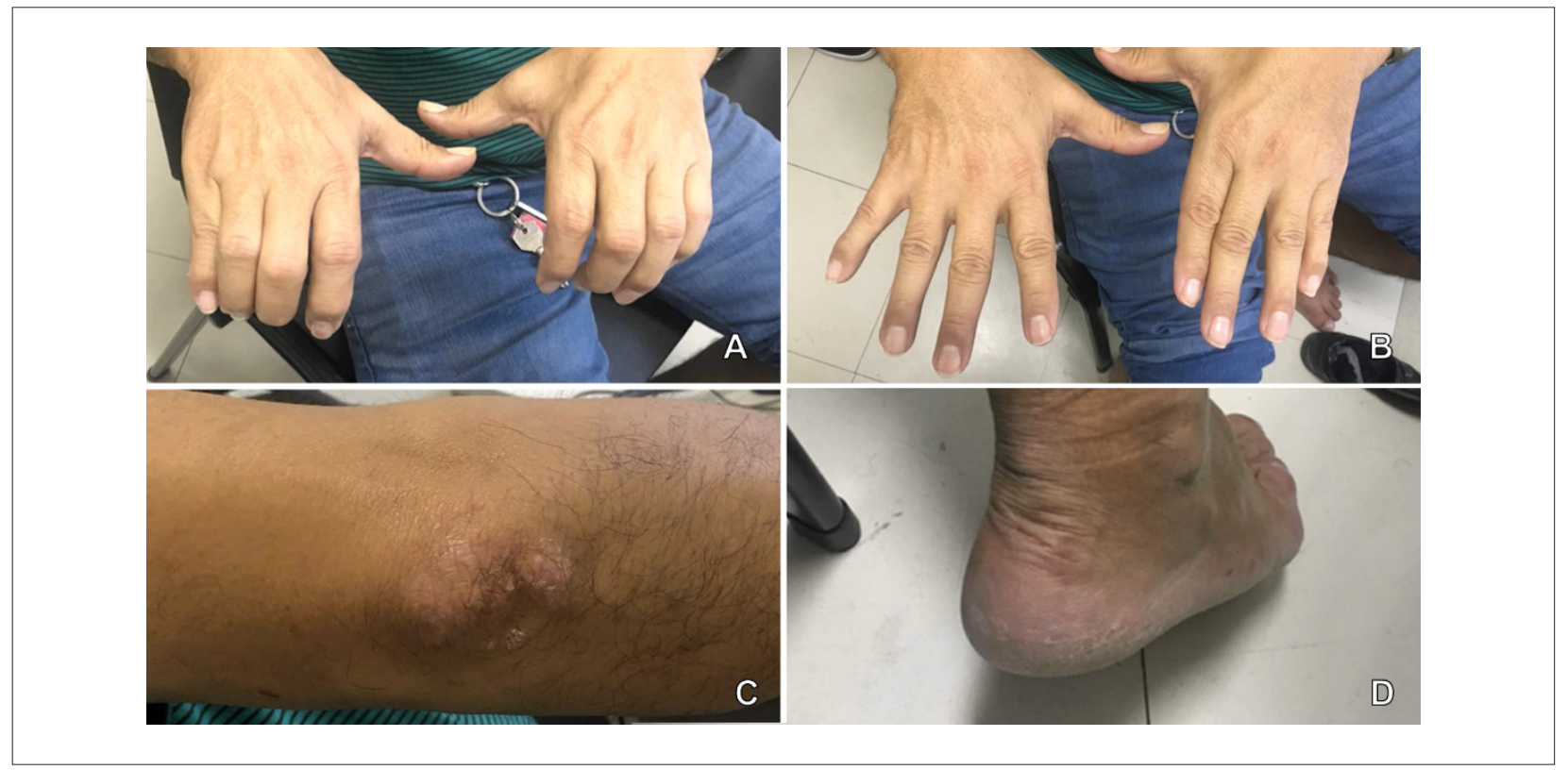

Figura 2 - Regressão dos xantomas. A e B) Articulações interfalângicas e metacarpofalângicas. C) Cotovelo direito. D. Região do tendão de Aquiles direito. Fonte: imagens obtidas pelos autores durante consulta de rotina.

no fenótipo I que no $\mathrm{V}^{2,6,14}$ Nesse caso, o diagnóstico de diabetes se deu após o relato de pancreatite, sugerindo hipertrigliceridemia relevante, por causa genética ou ambiental, pois o paciente não era totalmente aderente ao uso de fibrato.

Nem sempre o fenótipo das dislipidemias mostra-se claro, mesmo com exames complementares, dificultando o diagnóstico precoce e a conduta apropriada. ${ }^{15}$
A associação de estatina (alta potência) e ciprofibrato alcançou o objetivo esperado, haja vista os resultados laboratoriais e a cicatrização dos xantomas. Caso não fossem alcançadas as metas de lipoproteína de baixa densidade (LDL-C), a associação de estatina com inibidores de pró-proteína convertase subtilisina/kexina tipo 9 (PCSK9) ou ezetimiba seria uma opção, assim como ômega-3, em conjunto com fibrato, para redução da hipertrigliceridemia. ${ }^{2}$ 


\section{Carta Científica}

\section{Tabela 1 - Exames laboratoriais}

\begin{tabular}{lcc}
\hline Exames laboratoriais & Anterior ao tratamento & Após tratamento \\
\hline Triglicérides & $2.407 \mathrm{mg} / \mathrm{dL}$ & $291 \mathrm{mg} / \mathrm{dL}$ \\
\hline Colesterol total & $513 \mathrm{mg} / \mathrm{dL}$ & $144 \mathrm{mg} / \mathrm{dL}$ \\
\hline HDL-c & $40 \mathrm{mg} / \mathrm{dL}$ & $36 \mathrm{mg} / \mathrm{dL}$ \\
\hline LDL-c & $\mathrm{NC}$ & $50 \mathrm{mg} / \mathrm{dL}$ \\
\hline Glicemia & $234 \mathrm{mg} / \mathrm{dL}$ & $137 \mathrm{mg} / \mathrm{dL}$ \\
\hline Hemoglobina glicada & $10 \%$ & $7,1 \%$ \\
\hline TGO & $12 \mathrm{U} / \mathrm{L}$ & $13 \mathrm{U} / \mathrm{L}$ \\
\hline TGP & $21 \mathrm{U} / \mathrm{L}$ & $7 \mathrm{U} / \mathrm{L}$ \\
\hline TSH & $4,27 \mathrm{mU} / \mathrm{L}$ & $3,62 \mathrm{mU} / \mathrm{L}$ \\
\hline CPK & $\mathrm{VI}$ & $74 \mathrm{U} / \mathrm{L}$ \\
\hline Creatinina & $0,7 \mathrm{mg} / \mathrm{dL}$ & $\mathrm{VI}$ \\
\hline Ácido úrico & $\mathrm{Vl}$ & $6,4 \mathrm{mg} / \mathrm{dL}$ \\
\hline
\end{tabular}

HDL: lipoproteína de alta densidade; LDL: lipoproteína de baixa densidade; NC: não calculado pela Fórmula de Friedewald; VI: valor indisponível; TGO: aspartato aminotransferase; TGP: alanina aminotransferase; TSH: hormônio estimulante da tireoide; CPK: creatinofosfoquinase. Fonte: revisão de prontuário pelos autores.

Como limitações, ressaltamos que, por indisponibilidade na instituição, não foram realizados: angiotomografia de coronárias, para melhor estratificar o risco cardiovascular, ${ }^{2,16}$ apesar de o paciente ser de alto risco, ${ }^{9}$ e testes genéticos, para avaliar possíveis mutações em lipase lipoproteica e apolipoproteína E. Apesar disso, a avaliação clínica e laboratorial, aliada à experiência do serviço, foi fundamental para o resultado satisfatório, evitando-se manifestação de desfecho aterosclerotíco ou de nova pancreatite. Embora cada vez mais presente, a genotipagem ainda não está amplamente disponível em muitos países e serviços. ${ }^{17,18}$

\section{Conclusão}

Mesmo com as dificuldades inerentes à investigação laboratorial, a expertise em detectar e tratar adequadamente caso raro e grave de dislipidemia foi fundamental para a melhora laboratorial e a prevenção de resultados clínicos potencialmente fatais.

\section{Contribuição dos autores}

Concepção e desenho da pesquisa, Obtenção de dados, Análise e interpretação dos dados, Redação do manuscrito e
Revisão crítica do manuscrito quanto ao conteúdo intelectual importante: Manfredini E, Alves RJ.

\section{Potencial conflito de interesse}

Não há conflito com o presente artigo

\section{Fontes de financiamento}

O presente estudo não teve fontes de financiamento externas.

\section{Vinculação acadêmica}

Não há vinculação deste estudo a programas de pósgraduação.

\section{Aprovação ética e consentimento informado}

Este estudo foi aprovado pelo Comitê de Ética da Santa Casa de Misericórdia de São Paulo sob o número de protocolo CAAE: 23019019.3.0000.5479. Todos os procedimentos envolvidos nesse estudo estão de acordo com a Declaração de Helsinki de 1975, atualizada em 2013. O consentimento informado foi obtido de todos os participantes incluídos no estudo.

\section{Referências}

1. Grundy SM, Stone NJ, Bailey AL, Beam C, Birtcher KK, Blumenthal RS, et al. 2018 AHA/ACC/AACVPR/AAPA/ABC/ACPM/ADA/AGS/APhA/ASPC/NLA/ PCNA Guideline on the Management of Blood Cholesterol: A Report of the American College of Cardiology/American Heart Association Task Force on Clinical Practice Guidelines. Circulation. 2019;139(25):1082-143. doi: 10.1161/CIR.0000000000000625.

2. Faludi AA, Izar MCO, Saraiva JFK, Chacra APM, Bianco HT, Afiune A Neto, et al. Atualização da Diretriz Brasileira de Dislipidemias e Prevenção da

Aterosclerose - 2017. Arq Bras Cardiol. 2017;109(2 Supl 1):1-76. doi: 10.5935/abc. 20170121.

3. Santos RD, Gagliardi AC, Xavier HT, Casella A Filho, Araújo DB, Cesena FY, et al. First Brazilian Guidelines for Familial Hypercholesterolemia. Arq Bras Cardiol. 2012;99(2 Suppl 2):1-28. doi: 10.5935/abc.20120202.

4. Fredrickson DS, Levy RI, Lees RS. Fat Transport in Lipoproteins An Integrated Approach to Mechanisms and Disorders. N Engl J Med. 1967;276(1):34-42. doi: 10.1056/NEJM196701052760107. 
5. Fredrickson DS, Lees RS. A System for Phenotyping Hyperlipoproteinemia. Circulation. 1965;31:321-7. doi: 10.1161/01.cir.31.3.321.

6. Hegele RA, Ginsberg HN, Chapman MJ, Nordestgaard BG, Kuivenhoven JA, Averna M, et al. The Polygenic Nature of Hypertriglyceridaemia: Implications for Definition, Diagnosis, and Management. Lancet Diabetes Endocrinol. 2014;2(8):655-66. doi: 10.1016/S2213-8587(13)70191-8.

7. Hopkins PN, Toth PP, Ballantyne CM, Rader DJ. Familial Hypercholesterolemias: Prevalence, Genetics, Diagnosis and Screening Recommendations from the National Lipid Association Expert Panel on Familial Hypercholesterolemia. J Clin Lipidol. 2011;5(3 Suppl):9-17. doi: 10.1016/j.jacl.2011.03.452.

8. Kate GJRT, Bos S, Dedic A, Neefjes LA, Kurata A, Langendonk JG, et al. Increased Aortic Valve Calcification in Familial Hypercholesterolemia: Prevalence, Extent, and Associated Risk Factors. J Am Coll Cardiol. 2015;66(24):2687-95. doi: 10.1016/j.jacc.2015.09.087.

9. Mach F, Baigent C, Catapano AL, Koskinas KC, Casula M, Badimon L, et al. 2019 ESC/EAS Guidelines for the Management of Dyslipidaemias: Lipid Modification to Reduce Cardiovascular Risk. Eur Heart]. 2020;41(1):111-88. doi: 10.1093/eurheartj/ehz455.

10. Keren Z, Falik-Zaccai TC. Cerebrotendinous Xanthomatosis (CTX): A Treatable Lipid Storage Disease. Pediatr Endocrinol Rev. 2009; 7(1):6-11

11. Nie S, Chen G, Cao X, Zhang Y. Cerebrotendinous Xanthomatosis: A Comprehensive Review of Pathogenesis, Clinical Manifestations, Diagnosis, and Management. Orphanet J Rare Dis. 2014;9:179. doi: 10.1186/s13023-014-0179-4.
12. Expert Panel on Integrated Guidelines for Cardiovascular Health and Risk Reduction in Children and Adolescents; National Heart, Lung, and Blood Institute. Expert Panel on Integrated Guidelines for Cardiovascular Health and Risk Reduction in Children and Adolescents: Summary Report. Pediatrics. 2011;128 (Suppl 5):213-56. doi: 10.1542/peds.2009-2107C.

13. Yoo EG. Sitosterolemia: A Review and Update of Pathophysiology, Clinical Spectrum, Diagnosis, and Management. Ann Pediatr Endocrinol Metab. 2016;21(1):7-14. doi: 10.6065/apem.2016.21.1.7.

14. Scherer J, Singh VP, Pitchumoni CS, Yadav D. Issues in Hypertriglyceridemic Pancreatitis: An Update. J Clin Gastroenterol. 2014;48(3):195-203. doi 10.1097/01.mcg.0000436438.60145.5a.

15. García-Giustiniani D, Stein R. Genetics of Dyslipidemia. Arq Bras Cardiol. 2016;106(5):434-8. doi: 10.5935/abc.20160074.

16. Pearson TA, Mensah GA, Alexander RW, Anderson JL, Cannon RO 3rd, Criqui $\mathrm{M}$, etal. Markers of Inflammation and Cardiovascular Disease: Application to Clinical and Public Health Practice: A Statement for Healthcare Professionals from the Centers for Disease Control and Prevention and the American Heart Association. Circulation. 2003;107(3):499-511. doi: 10.1161/01. cir.0000052939.59093.45.

17. Stein R, Ferrari F, Scolari F. Genetics, Dyslipidemia, and Cardiovascular Disease: New Insights. Curr Cardiol Rep. 2019;21(8):68. doi: 10.1007/ s11886-019-1161-5.

18. Berberich AJ, Hegele RA. The Role of Genetic Testing in Dyslipidaemia. Pathology. 2019;51(2):184-92. doi: 10.1016/j.pathol.2018.10.014. 